\title{
Cytoplasmic RNA Granules in Somatic Maintenance
}

\author{
Ossama Moujaber Ursula Stochaj \\ Department of Physiology, McGill University, Montreal, QC, Canada
}

\section{Keywords}

Protein droplet $\cdot$ Neuronal granules $\cdot \mathrm{P}$ bodies $\cdot$ Stress granules

\begin{abstract}
Cytoplasmic RNA granules represent subcellular compartments that are enriched in protein-bound RNA species. RNA granules are produced by evolutionary divergent eukaryotes, including yeast, mammals, and plants. The functions of cytoplasmic RNA granules differ widely. They are dictated by the cell type and physiological state, which in turn is determined by intrinsic cell properties and environmental factors. RNA granules provide diverse cellular functions. However, all of the granules contribute to aspects of RNA metabolism. This is exemplified by transcription, RNA storage, silencing, and degradation, as well as mRNP remodeling and regulated translation. Several forms of cytoplasmic mRNA granules are linked to normal physiological processes. For instance, they may coordinate protein synthesis and thereby serve as posttranscriptional "operons". RNA granules also participate in cytoplasmic mRNA trafficking, a process particularly well understood for neurons. Many forms of RNA granules support the preservation of somatic cell performance under normal and stress conditions. On the other hand, severe insults or disease can cause the formation and persistence of RNA
\end{abstract}

granules that contribute to cellular dysfunction, especially in the nervous system. Neurodegeneration and many other diseases linked to RNA granules are associated with aging. Nevertheless, information related to the impact of aging on the various types of RNA granules is presently very limited. This review concentrates on cytoplasmic RNA granules and their role in somatic cell maintenance. We summarize the current knowledge on different types of RNA granules in the cytoplasm, their assembly and function under normal, stress, or disease conditions. Specifically, we discuss processing bodies, neuronal granules, stress granules, and other less characterized cytoplasmic RNA granules. Our focus is primarily on mammalian and yeast models, because they have been critical to unravel the physiological role of various RNA granules. RNA granules in plants and pathogens are briefly described. We conclude our viewpoint by summarizing the emerging concepts for RNA granule biology and the open questions that need to be addressed in future studies.

(c) 2018 S. Karger AG, Basel

\section{What Are RNA Granules?}

We define RNA granules as spherical membrane-less compartments that are composed of RNA and proteins. Intracellular RNA granules are diverse; they differ in lo-

\section{KARGER}

(c) 2018 S. Karger AG, Basel

E-Mail karger@karger.com

www.karger.com/ger
Ursula Stochaj

Department of Physiology, McGill University

3655 Promenade Sir-William-Osler

Montreal, QC H3G 1 Y6 (Canada)

E-Mail ursula.stochaj@ mcgill.ca 
cation, composition, and size. There is no consensus on the size constraints, but most RNA granules described to date have been visualized by optical microscopes, including recent super-resolution techniques. The size of nucleoli and stress granules (SGs) can amount to several micrometers [1-3]. By contrast, mRNA processing bodies (P bodies, PBs) $[4,5]$ and other RNA granules are often much smaller (see below).

The formation of granular compartments offers several advantages over membrane-enclosed organelles. As such, RNA granule biogenesis is highly flexible and supports rapid adaptive responses to changes in cell physiology. Moreover, the lack of membrane barriers facilitates the fast exchange with surrounding cellular components, even for large molecules [6].

Most RNA granules operate like droplets or hydrogels $[7,8]$, a property provided by RNA-binding proteins that contain low complexity or disordered domains. These regions favor protein self-association and binding to other polypeptides with similar domains $[6,9,10]$. Granule formation is further promoted by RNA-protein interactions [11]. Together, these associations generate dynamic compartments that concentrate selected factors in a highly localized fashion [6] and contribute to various aspects of RNA metabolism. Particularly, RNA granules synthesize, modify, protect, store, release, transport, and degrade RNA in a regulated fashion $[9,11]$.

\section{RNA Granules: Location, Composition, and Size Matter}

Eukaryotic cells assemble RNA granules in different subcellular locations; they are present in the cytoplasm and several subcellular organelles, including nuclei, mitochondria, and chloroplasts. Granule functions are dictated by subcellular location, macromolecular composition, and the physiological state of the cell. In line with their distinct activities, RNA granule biogenesis and dynamics vary considerably. Some RNA granules are present under all growth conditions, whereas others are induced by stress.

\section{RNA Granule Subcellular Location}

RNA granules in nuclei are also referred to as nuclear bodies. Nuclear bodies regulate cell growth and survival, proliferation, gene expression, and splicing. Nucleoli are the most prominent RNA granules in the nucleus; they assemble ribosomes, assist in genome maintenance and facilitate DNA repair. Moreover, nucleoli control stress responses, cell senescence, and apoptosis [12]. Thus, they are indispensable for the maintenance of nucleated somatic cells. Other nuclear RNA granules are also required for normal growth or during stress. For example, nuclear speckles contain RNA-processing enzymes and accelerate heat-induced chaperone gene expression [13]. Although separated by the nuclear envelope, nuclear and cytoplasmic RNA granules can share protein components and may communicate [14]. As nuclear bodies have been discussed in detail $[12,15,16]$, we focus here on somatic RNA granules in the cytoplasm and their roles in cellular and tissue maintenance.

Cytoplasmic granule distribution is often dynamic and relies on interactions with the cytoskeleton. Different filament systems engage with RNA granules [17]. Many RNA granules associate with microtubules via various motor proteins. This promotes granule movement, fusion, disassembly, and exchange of RNA or protein between granules or with the surrounding cytoplasm. Binding to actin bundles can generate stationary granules in mammals, but actin filaments mediate granule movement in plants.

\section{RNA Granule Composition}

Cytoplasmic RNA granules are heterogeneous mixtures of proteins and nucleic acids [18]. RNA-binding proteins regulate granule assembly and properties. This is well established for proteins associating with mRNA, as they determine mRNA fate from transcription to protein synthesis. So far, more than 1,000 proteins are known to interact with mRNA. These associations occur with distinct stoichiometries and affinities [18]; they involve three major mRNA domains. The $5^{\prime}$-untranslated region (UTR) has relatively little protein bound, while the open reading frame $(\mathrm{ORF})$ interacts with proteins that regulate translation (example: FMR1, see below). The lengths of $3^{\prime}$-UTRs vary widely and can be organ or cell-type specific. In general, $3^{\prime}$-UTRs are longer in the brain. This is relevant, because $3^{\prime}$-UTRs bind numerous proteins (examples: FUS, HuR, Ago) and thereby generate a diverse collection of mRNP granules.

Despite this complexity, marker proteins can identify specific RNA granule types. At the same time, there is extensive cross-talk between granular compartments. Thus, when in close proximity, RNA granules often exchange macromolecules (reviewed in [19]). Moreover, distinct granule types can overlap in composition, as illustrated by PBs and SGs [20], or yeast EGP bodies and SGs [21]. On the other hand, some RNA granules are divided into subclasses. This is exemplified by SGs, for which the 
stressor determines granule size and protein make-up ([3] and below).

The presence of diverse RNA species further expands granule diversity. Aside from mRNA, this includes various noncoding RNAs, such as ribosomal RNA, microRNA, and long noncoding RNA [2, 22, 23]. While mRNAs can move between different granules [24], the intergranular trafficking of noncoding RNAs is poorly defined.

\section{Granule Size}

RNA granules differ profoundly in size, ranging from several micrometers for SGs to $\geq 100 \mathrm{~nm}$ for PBs [3]. Different macromolecular associations stimulate $\mathrm{PB}$ assembly, and the sum of these interactions determines the final granule dimensions [5]. This principle of tunable size likely applies to other RNA granules as well. For instance, the loss of SG nucleating proteins during cell senescence profoundly changes granule size [25].

\section{Cytoplasmic RNA Granule Properties and Control of Somatic Cell Homeostasis}

Overall, RNA granules are involved in RNA quality control and metabolism. However, cytoplasmic RNA granules differ markedly in size, composition, and mode of biogenesis. Accordingly, they contribute to various aspects of cellular homeostasis. Some granules, such as PBs and SGs, are assembled by many cell types and organisms, suggesting more general functions for cellular maintenance. Other RNA granules may be limited to certain cell types or organisms, for instance neurons or pathogens (online suppl. Table; for all online suppl. material, see www.karger.com/doi/10.1159/000488759). Moreover, RNA granule formation and dynamics are sensitive to changes in cell physiology and signaling events. While some RNA granules are formed under all growth conditions, others assemble in response to stress (Fig. 1). Stressors can be endogenous (such as nutrient starvation) or environmental (heat).

\section{RNA Granules Generated in the Absence of Stress}

\section{$P$ Bodies}

Based on electron microscopy, the diameter of mammalian PBs varies between 150 and $340 \mathrm{~nm}$ [4]. Nanoparticle tracking analysis determined the size of yeast PBs as $\sim 380 \mathrm{~nm}$ in wild-type cells [5]. Several redundant mechanisms drive PB assembly, at least in yeast [5]. Studies in

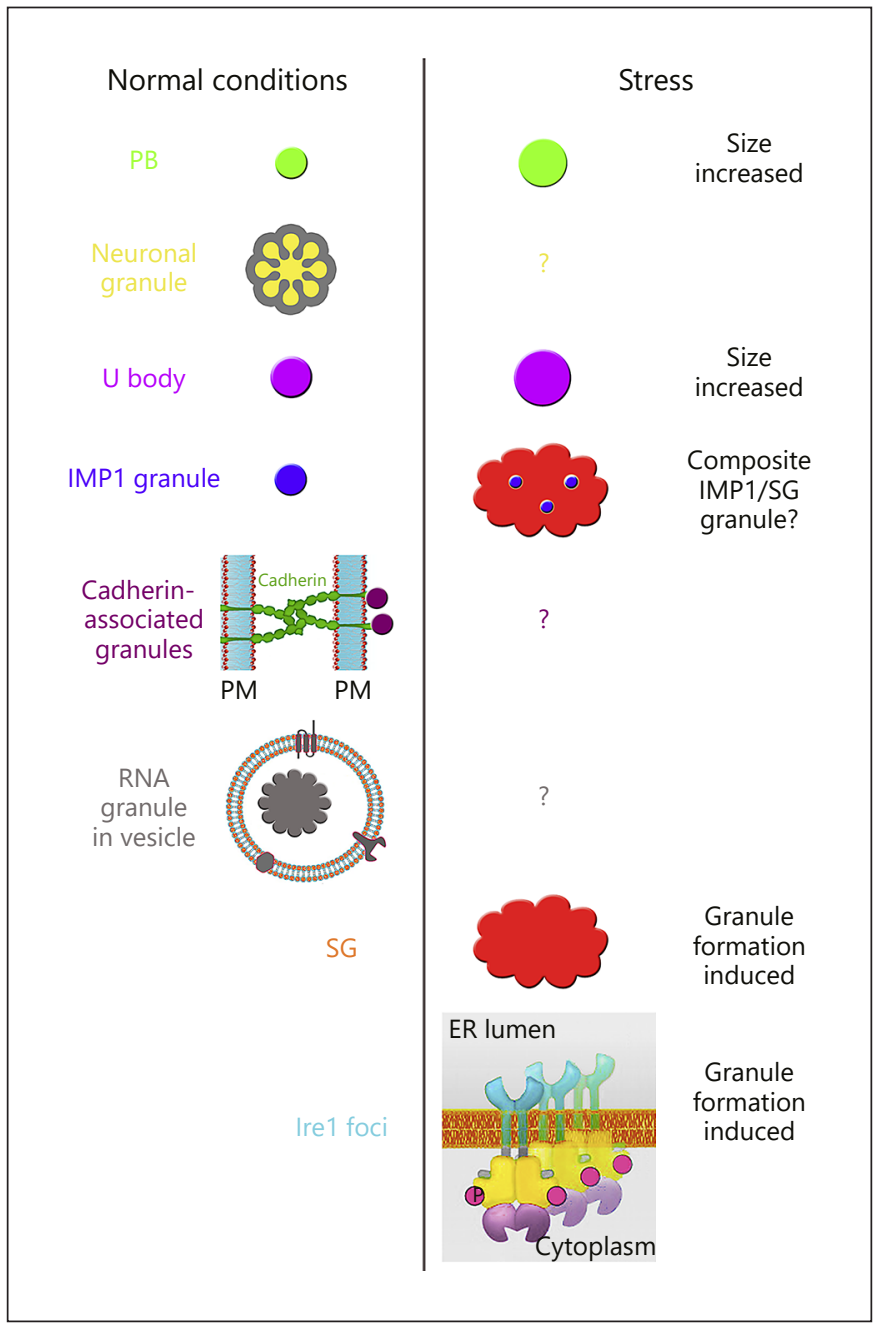

Fig. 1. Cytoplasmic RNA granules assemble under normal and stress conditions. The fate of RNA granules during stress is not always fully defined, as indicated by the question marks. Some granules, such as PBs and U bodies, increase in size during stress. Membrane-associated RNA granules are present in epithelial cells at the zonula adherens. RNA granules are also sequestered into vesicles that can be part of cytoplasmic multivesicular bodies. Under stress conditions, SGs and Ire1 foci form; they are absent in nonstressed cells. Ire 1 activation leads to unconventional splicing of Xbp $1 \mathrm{mRNA}$ in the cytoplasm. Activated Ire1 also promotes the degradation of mRNAs that are translated at the ER membrane. The depiction of Ire1 foci was adapted from [26]. PM, plasma membrane. See text for details.

the yeast Saccharomyces cerevisiae and mammalian cells link PB proteins to various aspects of RNA homeostasis. These include transcription, splicing, mRNA trafficking, cap-binding, decapping, deadenylation and degradation, nonsense-mediated decay, RNP remodeling, gene silencing, translation activation and inhibition via miRNAs, 
and other mechanisms [27-29]. PBs also contain protein kinases, ubiquitin ligases, and components limiting virus replication. Structurally, PBs are divided into two different subcompartments. A shell with polysomes and translational activators surrounds a dense core with mRNA decay factors [4]. This is consistent with the idea that transcripts in the shell can re-enter translation.

The advantages of PB granule production are not fully understood. Deadenylation and translational repressors are required for $\mathrm{PB}$ assembly [20, 30, references therein]. Deadenylation is also needed for mRNA decay in mammalian cells [30]. On the other hand, mRNA decapping occurs in the absence of $\mathrm{PB}$ granule production. These observations are united in a model that describes $\mathrm{PB}$ assembly as a consequence, not a cause, of mRNA repression and degradation [31]. Aside from the concentrating factors involved in mRNA metabolism, granule building could also enhance and coordinate the cytoplasmic trafficking of these factors [17].

A recent study in HEK293 cells identified 125 proteins of the PB proteome [20]. Approximately half of these proteins interact with the helicase DDX6, a PB resident that is present in multiple protein complexes [20,29]. About $20 \%$ of cytoplasmic transcripts concentrate in PBs, and these RNAs are enriched for protein-coding transcripts. Interestingly, PBs have only a limited role in mRNA degradation, at least in mammalian cells [20]. By contrast, they provide foci that control the translation of mRNAs that encode regulatory switches. Specific examples include components involved in chromatin remodeling or cell cycle regulation. The condensation of mRNPs into granules offers a powerful mechanism to coordinate the translation of pathway-related transcripts. This could be achieved through (i) the segregation of translationally repressed mRNAs and (ii) their simultaneous release from PBs. Whether the spatial proximity between PBs and ribosomes or mitochondria [4] contributes to this regulation remains to be determined.

Mammalian cell culture models and the yeast S. cerevisiae provided insights into the physiological roles of PBs. As such, PB proteins control neuronal differentiation during the withdrawal of nerve growth factor [32]. In this scenario, $\mathrm{PB}$ components are needed to downregulate miRNA activity, which ultimately supports neuronal survival during growth factor withdrawal.

In budding yeast, $\mathrm{PB}$ proteins are critical for genomic stability and the response to DNA replication stress [33, 34]. Genomic stability relies on the proper stoichiometry of histones [33], which is controlled by Lsm1. Concentrated in PBs, Lsm 1 stimulates the degradation of histone
mRNA, which limits the abundance of histones and thereby diminishes DNA replication stress. While the decay of histone mRNA is likely located in PBs, it should be noted that formally this has not been shown [33]. Additional evidence emphasizes the importance of PB proteins for DNA replication [34]. Specifically, the loss of Lsm1 leads to excessive rewiring of the transcriptome during DNA replication stress and makes cells more vulnerable to the insult. Dysfunctional PBs in $l s m 1$ mutant cells cause this increased susceptibility. The mutant cells cannot limit the abundance of the transcriptional repressor Yox1, likely because PBs fail to repress properly the translation of Yox1 mRNA. Interestingly, the circadian clock controls PB formation [35]. Thus, it is possible that DNA repair or regulatory switches associated with $\mathrm{PBs}$ undergo rhythmic changes.

\section{RNA Granules in Neurons}

Developing and adult neurons translate mRNAs in multiple subcellular locations. Aside from the cell soma, protein synthesis also occurs in axons and dendrites. The local translation of mRNAs in neurites is essential for neuronal development, growth cone and synapse formation, cell survival, maintenance, and function, including neuronal plasticity, memory, and learning $[36,37$, references therein]. The axons of developing retinal ganglion cells contain two major mRNA groups: (1) constitutively translated transcripts for energy and protein homeostasis; (2) transcripts translated in a developmental stagedependent fashion. The second group is relevant to axon elongation, branching, pruning, and synapse formation. Moreover, adult neurons control axon survival and neurotransmission by localized translation.

In many cases, locally translated mRNAs are present in RNA granules. Overall, the size of neuronal granules varies from 300 to $1,000 \mathrm{~nm}$. However, it is possible, that larger granules are composites and comprised of smaller entities, each $100-300 \mathrm{~nm}$ in size. Proteins and mRNAs associated with neuronal granules have been identified [18, 36-39]. Together with other data, these studies support several key concepts. (i) Locally translated mRNAs carry common sequence elements that promote their subcellular distribution. These sequence elements are often present in the $3^{\prime}$-UTR, which is generally longer in brain cells. (ii) The composition of neuronal granules is highly diverse. Granule-associated mRNAs change during development. (iii) Ribosomal proteins are abundant in neuronal granules. RNA-binding proteins in neuronal granules show some overlap with other RNA granules (examples: G3BP1, Staufen1). However, neuronal granules are en- 
riched for a subset of RNA-binding proteins, including Fragile X protein (FMRP) and FMRP-related proteins (FXR1P and FXR2P). (iv) During transport to neurites granule mRNAs are stalled in translation. This reversible stalling is mediated by translational repressors. (v) Proteins associated with the mRNA control the translational reactivation at the final granule destination in dendrites or axons. FMRP, Staufen, and UPF1 are key players that control the repression and reactivation of mRNA translation in neurites. (vi) Posttranslational modifications of mRNAbinding proteins, such as FMRP phosphorylation, can provide a switch to reverse translational inhibition. (vii) Local translation can be constitutive, developmentally regulated, or initiated by specific signaling events. For instance, the activation of synaptic metabotropic glutamate receptors (mGluRs) leads to FMRP dephosphorylation, reactivation of translation, and the synthesis of proteins involved in mGluR long-term repression [37, references therein]. Aside from long-term depression, granule-associated proteins also impinge on long-term potentiation, learning, and memory (online suppl. Table). (viii) Neuronal granules assemble initially in the soma. Given the limited width of dendritic spine necks, they may travel as small granules that fuse after reaching their final destination. (ix) Neuronal granule transport is mediated by microtubules, but granules also associate with other cytoskeletal elements, including neurofilaments. (x) In addition to "conventional" neuronal RNA granules, neurons contain large megaRNPs, formed by nuclear envelope budding [40]. MegaRNPs are present in multiple cell types; in neurons, they contain a limited collection of mRNAs and play a role for synaptic plasticity.

Given the importance of RNA granules for neuronal development, maintenance, and activity, it is not surprising that dysfunctional neuronal granule proteins are linked to human diseases. These include the wide spectrum of amyotrophic lateral sclerosis-frontotemporal dementia and other disorders ([3, 41], see also online suppl. Table).

\section{IMP1 (ZBP1) Granules}

In mammalian cells, insulin-like growth factor 2 mRNA-binding proteins (IGF2BPs, IMPs) are present in cytoplasmic IMP1 granules [42, 43]. IMP1, also known as zip-code-binding protein 1 (ZBP1), regulates $\beta$-actin mRNA localization by binding a $3^{\prime}$-UTR motif. IMP1 granules are 100-300 $\mathrm{nm}$ in diameter and composed of IMPs, 40S ribosomal subunits, hnRNPs, polyA-binding proteins, and mRNA. In HEK293 cells, IMP1 granules contain $\sim 3 \%$ of the transcriptome. Granule-associated

Cytoplasmic RNA Granules in Somatic

Maintenance transcripts are enriched for components of the secretory pathway, ubiquitin-dependent protein degradation, mRNA processing and translation. IMP granules differ from PBs, SGs, and Staufen-containing mRNA granules, but may be related to other neuronal granules. As eIF4E, eIF4G, and 60 S ribosomal subunits are missing, IMP1 granules do not support translation, but may control pathway-dependent gene expression. Indeed, IMP1 granules stabilize mRNAs encoding high molecular weight Tau in PC12 cells [43]. During arsenite stress, a portion of IMP1 colocalizes with G3BP1 in SGs.

\section{U Bodies}

The nuclear RNA processing machinery contains uridine-rich small RNAs and proteins that combine to ribonucleoprotein particles (U snRNPs). Most of these particles are assembled in the cytoplasm [44, references therein]. U bodies represent $200-400 \mathrm{~nm}$ spherical compartments. They concentrate U snRNPs and factors required for their assembly, including the survival motor neuron (SMN) protein complex. U bodies are physically connected to PBs and the ER; they assemble and/or store U snRNPs. Nutritional and heat stress can enlarge U bodies [44], possibly due to the stress-induced increase in snRNP storage. The SMN protein is one of the components shared by $\mathrm{U}$ bodies and neuronal granules.

\section{Translationally Active mRNA Granules}

In $S$. cerevisiae, most mRNAs are distributed diffusely throughout the cytoplasm. However, selected mRNAs locate in granules that are translationally active in the absence of stress. Granule formation may orchestrate metabolic control through mRNA localization and the coordinated synthesis of proteins involved in the same pathway. Upon glucose or amino acid starvation, these granules coalesce into larger compartments, some of which resemble SGs (see below) [45]. During glucose depletion, granule fusion is accompanied by the recruitment of $\mathrm{PB}$ components.

\section{Cadherin-Associated RNA Granules}

Epithelial cells contain spherical RNA-protein compartments at the apical zonula adherens [46]. Cadherin complexes are associated with active RISC (RNA-induced silencing complex) and a subset of mRNAs, which are often linked to cell stemness, Wnt/ $\beta$-catenin or TGF- $\beta$ pathways. The recruitment of RISC and selected mRNAs to the apical zonula adherens could link the junctional integrity to signaling routes that are important for growth and homeostasis of epithelial cells. 


\section{RNA Granules in Mitochondria}

Mitochondrial RNA granules (MRGs) are located in the mitochondrial matrix, where they contribute to the proper expression of mitochondrial genes. Several MRGassociated proteins were identified and possible functions in posttranscriptional events have been uncovered [47, 48, references therein]. MRGs are implicated in multiple activities, including RNA processing, RNA modification (methylation, pseudouridylation), RNA degradation, and the assembly of mitochondrial ribosomes. The heterogeneity in composition and spatial distribution could explain the functional diversity of MRGs. For instance, about $10-20 \%$ of MRGs are in close proximity to nucleoids, where they may be involved in the initial processing of mitochondrial polycistronic transcripts [47].

MRG formation depends on the presence of RNA, and the granules are likely dynamic. To date, it is unknown whether MRG form droplets or hydrogels (see above).

\section{RNA Granules in Multivesicular Bodies}

RNA granules have also been detected in multivesicular bodies or exosomes (online suppl. Table). Following the secretion of the vesicle, enclosed mRNPs may facilitate a systemic exchange of miRNAs and other RNAs.

\section{Stress-Induced RNA Granules}

\section{Stress Granules}

Many endogenous or exogenous stressors cause the stalling of mRNA translation initiation and the subsequent assembly of cytoplasmic SGs. These granules are organized into cores and surrounding shells. They contain mRNA, RNA-binding proteins, and components of the $40 \mathrm{~S}$ ribosomal subunit (reviewed in $[3,8,49,50]$ ). Determined by the duration and type of stress as well as granule composition, SG diameters range from 0.1 to $4 \mu \mathrm{m}[3,8]$.

Based on their mode of assembly, SGs fall into two major subclasses. Canonical SGs (example: arsenite SGs) require the phosphorylation of eukaryotic translation initiation factor eIF2 $\alpha$. By contrast, noncanonical SGs (example: pateamine A SGs) form independent of eIF2a phosphorylation. SG production begins with cytoplasmic nucleating foci that coalesce and mature through the addition of granule material. Nucleating foci can be produced by the RNA-binding protein TIA (T-cell intracellular antigen 1) or G3BP1 (Ras-GAP SH3 binding protein 1 [51]). Similar to other RNA granules, microtubules are critical for SG fusion and disassembly $[3,8,49,50]$. Aside from proteins, SGs contain different RNA species [22]. More than $78 \%$ of the RNAs in SG cores represent mRNA, but noncoding RNAs are also present. In U-2 OS cells (human osteosarcoma), $10-13 \%$ of the total mRNA locates in arsenite-induced SGs. Three mRNA properties favor SG core localization: ineffective translation, lack of membrane association, long coding regions and long $3^{\prime}$ UTRs [22]. However, high efficiency transcript concentration in granules ( $>50 \%$ of mRNA molecules in SGs) occurred for only 185 genes.

Molecular chaperones perform SG quality control, called granulostasis [52]. Autophagy and chaperone function are crucial for SG disassembly when stress subsides. To date, little is known about the quality control for SGlocated RNAs.

Multiple links connect SGs to the recovery or maintenance of cellular homeostasis under stress. (i) SGs accumulate mRNAs stalled in translation initiation. This stalling limits energy consumption under acute stress. Granule-associated mRNAs can resume translation or undergo degradation. (ii) In general, mRNA stabilization does not require SG formation [53]. Nevertheless, long transcripts may be better preserved by sequestration into granules. (iii) SGs promote the survival of stressed cells by several mechanisms. To this end, they limit the production of reactive oxygen species and sequester proapoptotic factors, thereby preventing cell damage and death.

Overall, SGs support cell survival during stress. Yet, SG formation can also be harmful when granulostasis is compromised. Under these conditions, SGs accumulate defective ribosomal products [52], which could contribute to neurodegeneration and other pathologies. Moreover, cell senescence compromises SG assembly and thus impairs the proper response to stress [25, 54].

At present, it is not known whether RNA sequestration in SGs benefits cellular homeostasis during stress. However, it is conceivable that SG association facilitates the coordinated mRNA release and translation when stress abates. Interestingly, many mRNAs encoding kinesintype motors are enriched in SGs. By contrast, various transcripts for proteins of cytoplasmic ribosomes are depleted from SGs (supplemental data in [22]). Whether this is relevant to cell physiology has to be addressed in future studies.

Compared to arsenite, SGs induced by other stressors are less well characterized (references in online suppl. Table). Prominent examples are granules assembled upon tRNA cleavage and chloroplast SGs (cpSGs). Various species generate tRNA fragments (tRFs) from mature or pretRNA. Cleavage can occur in response to nutrient starva- 
tion, and tRFs derived from the $5^{\prime}$-half of the tRNA cause $S G$ formation. Some tRFs also promote translational repression; this may depend on their ability to produce Gquadruplex structures.

Following oxidative stress, the green alga Chlamydomonas reinhardtii forms SGs in chloroplasts. In addition, C. reinhardtii chloroplasts generate granules that contain oxidized RNA. Arsenite-treated HeLa cells also produce cytoplasmic granules that accumulate oxidized RNA. These granules are clearly distinct from SGs and PBs. Their protein or RNA composition is not yet defined, but they could participate in RNA quality control [55, references therein].

\section{EGP Bodies}

Glucose starvation, a mild stressor, triggers the assembly of granules in yeast that concentrate translation initiation factors eIF4E, eIF4G, and polyA-binding protein Pab1p [21]. These EGP bodies lack RNA decay and decapping enzymes. They may store mRNA during exposure to specific stressors [21] and help re-establish translation when the stress subsides. How EGP bodies relate to translationally active mRNA granules (see above) is not clear.

\section{Ire1 Foci}

ER stress triggers clustering of the bifunctional endoribonuclease/protein kinase Ire1 at the ER membrane [26, 56]. Upon activation, Ire1 foci perform the unconventional splicing of Haclp/Xbpl mRNA, which encodes a stress-related transcriptional regulator. Ire1p also facilitates the degradation of mRNAs associated with the ER. Although less complex in composition and function than other RNA granules, Ire1 foci are clearly important for the survival of ER stress.

\section{RNA Granules and Aging}

SG formation is impaired in senescent cells $[25,54]$. At the organismal level, RNA-binding proteins are more likely to aggregate in Caenorhabditis elegans when the nematode ages [57]. This age-related tendency to aggregate includes PAB-1 and TIAR-2, homologs of the human SG proteins PABP-1 and TIA-1. In aged worms, PAB- 1 and TIAR- 2 concentrate in foci, where both proteins have low mobility. Thus, old animals form SG-related - but less dynamic - RNA granules, which may become persistent. Notably, the age-associated presence of large foci containing RNA-binding proteins correlates

Cytoplasmic RNA Granules in Somatic

Maintenance with a loss of organismal fitness. Interestingly, diminished signaling through insulin/insulin-like growth factor-1 receptor maintains the solubility of RNA-binding proteins [57]. The underlying molecular mechanisms are not fully defined.

\section{RNA Granules in Pathogenic Parasites}

The life cycles of Apicomplexa (several Plasmodium strains cause malaria in humans, Toxoplasma gondii toxoplasmosis) and Trypanosomatidae (Trypanosoma brucei - sleeping sickness; T. cruzi - Chagas disease; Leishmania - leishmaniasis) necessitates the switch of host organisms [58]. This requires the rapid adjustment to new environments and is accompanied by stress for the parasite. The ensuing adaptive changes in gene expression heavily rely on posttranscriptional mechanisms that involve SGs and other RNA granules. Since RNA granules are relevant to parasite survival, granule-associated components could emerge as new therapeutic targets.

\section{The Role of RNA Granules for Virus Infection and Cancer}

\section{Stress Granules}

SG formation is a survival strategy that protects cells from stress. Animal and plant cells also use these pathways to fight virus infections (online suppl. Table). At the same time, some viruses impair SG formation to escape host defense mechanisms or prevent the death of the host cell.

SG formation during cancer therapy has been reviewed recently $[3,59]$. In brief, cancer cells can produce SGs to survive some treatment regimens. Under these conditions, therapeutic inhibition of SG assembly may enhance the elimination of tumor cells.

\section{Anaplastic Lymphoma Kinase Granules}

Anaplastic large-cell lymphomas that produce nucleophosmin-anaplastic lymphoma kinase (NPM-ALK) fusions with ALK kinase activity assemble ALK granules [60]. ALK granules are $200-500 \mathrm{~nm}$ in diameter; they contain ALK, polyA-RNA, and RNA-binding proteins. ALK granules resemble SGs. However, they are present in nonstressed but transformed cells. ALK granules may enhance the translation of selected mRNAs by directing them to polysomes. This could boost cancer cell proliferation and survival. 


\section{Small MLN51-Induced Granules}

Metastatic lymph node 51 (MLN51) is a component of the exon-junction complex [18] that associates with mRNA after splicing. MLN51 is also involved in SG formation and present in PBs. Small MLN51-induced granules (SMIGs) form upon MLN51 overexpression; they contain RNAs and show directed movements. MLN51 overexpression causes $\mathrm{PB}$ disassembly. As some types of cancer overexpress MLN51, their PB formation will be impaired with possible consequences for the miRNA pathway.

\section{Conclusions}

RNA granules in the cytoplasm are involved in all aspects of RNA homeostasis. Their functional disparity is reflected by variations in localization, size, protein and RNA composition. Cell type-specific repertoires of RNA and RNA-binding proteins facilitate the assembly of RNA granules with divergent properties. Nevertheless, several cytoplasmic RNA granules display common features. Aside from their physicochemical properties (droplets, hydrogels), this may include tunable size, a concept initially developed for PBs.

Common principles could also underlie RNA granule function. For instance, PBs and neuronal granules control the activities of RNA "regulons", thereby orchestrating the expression of pathway-related mRNAs [61]. This property is likely shared by IMP1 granules, translationally active mRNA granules, cadherin-associated RNA granules, and ALK granules. For all of these compartments, the concentration of functionally connected RNAs supports the model that RNA granules operate as post-transcriptional operons. Moreover, as many RNA granules are mobile, they enable the transport of selected RNA or protein subsets in a highly coordinated fashion.

While RNA granule assembly is key to the maintenance or restoration of cellular homeostasis, impairment of RNA granule performance often has dire consequences for human health. The loss of granulostasis, including failure to dissolve granules, can lead to a broad range of neurodegenerative diseases. At the same time, RNA granule production during cancer therapy or by parasites can sustain the proliferation of tumor cells or pathogens.

The understanding of RNA granule properties and functions has progressed profoundly in recent years. Yet, there are still significant knowledge gaps related to even
Box 1. Open questions

Recent progress in the field of RNA granules has led to important new insights. Nevertheless, many topics require additional and comprehensive studies. We list some of the subjects that need to be investigated thoroughly in the future.

How does aging or senescence affect the assembly and features of different RNA granules? Can altered RNA granule properties accelerate aging?

Are there cell type- or organ-specific changes that impact RNA granules during aging?

Which of the RNA granules control the translation of RNA regulons? How is pathway-specificity achieved? Do RNAs of a regulon have common binding proteins?

Is RNA degradation controlled by RNA granules in a regulon-specific fashion?

Aside from Ire1 foci, multicellular vesicles, and possibly megaRNPs - which other RNA granules associate with membranes?

Are all RNA granules organized into cores and shells?

Do cytoplasmic RNA granules contain lipids? Do they play a role for cell signaling, granule assembly or dissolution?

RNA granules locate at the ER membrane (Ire1 foci) or are secreted via extracellular vesicles. To which extent do membrane-bound or secreted RNA granules regulate cellular and systemic homeostasis? Do cadherin-associated and vesicle-enclosed RNPs interact directly with the lipid bilayer?

Crosstalk takes place between PBs and SGs. How much crosstalk occurs between other cytoplasmic RNA granules? How is it regulated?

How do cytoplasmic RNA granules communicate with RNA granules in the nucleus, mitochondria, or chloroplast?

In addition to PBs, do circadian rhythms impinge on the assembly and function of other cytoplasmic RNA granules?

fundamental granule features. As such, RNA granules can locate in proximity to lipid droplets $[62,63]$, and lipid biosynthesis is potentially linked to $\mathrm{PB}$ formation [64]. To date, little is known about the presence of lipids in RNA granules. As some lipids have surfactant properties, they could impact the fusion and fission of RNA granules or control granule-dependent signaling events. Similarly, whether RNA granules contain complex carbohydrates is unclear.

Despite the importance of RNA granules for cellular maintenance, their aging-associated changes in organization and function are poorly understood. This is particularly relevant to human health, because the loss of 
neuroplasticity and many neurodegenerative diseases are linked to aging. These and other questions (see Box 1) remain to be addressed to fully appreciate the physiological and pathological roles of cytoplasmic RNA granules.

\section{Acknowledgement}

We thank D. Abou Samhadaneh for critical reading of the manuscript. This work was supported by grants from Natural Sciences and Engineering Research Council of Canada (NSERC). O.M. is the recipient of a McGill University Health Centre (MUHC) Graduate Studentship.

\section{References}

1 Hernandez-Verdun D: The nucleolus: a model for the organization of nuclear functions. Histochem Cell Biol 2006;126:135-148.

$\checkmark 2$ Anderson P, Kedersha N: RNA granules. J Cell Biol 2006;172:803-808.

-3 Mahboubi H, Stochaj U: Cytoplasmic stress granules: dynamic modulators of cell signaling and disease. Biochim Biophys Acta 2017; 1863:884-895.

4 Cougot N, Cavalier A, Thomas D, Gillet R: The dual organization of $\mathrm{P}$-bodies revealed by immunoelectron microscopy and electron tomography. J Mol Biol 2012;420:17-28.

5 Rao BS, Parker R: Numerous interactions act redundantly to assemble a tunable size of $\mathrm{P}$ bodies in Saccharomyces cerevisiae. Proc Natl Acad Sci USA 2017;114:E9569-E9578.

-6 Kaganovich D: there is an inclusion for that: material properties of protein granules provide a platform for building diverse cellular functions. Trends Biochem Sci 2017;42:765776.

7 Zhu L, Brangwynne CP: Nuclear bodies: the emerging biophysics of nucleoplasmic phases. Curr Opin Cell Biol 2015;34:23-30.

-8 Panas MD, Ivanov P, Anderson P: Mechanistic insights into mammalian stress granule dynamics. J Cell Biol 2016;215:313-323.

-9 Aguilera-Gomez A, Rabouille C: Membranebound organelles versus membrane-less compartments and their control of anabolic pathways in Drosophila. Dev Biol 2017;428:310317.

10 Protter DSW, Rao BS, Van Treeck B, Lin Y, Mizoue L, Rosen MK, Parker R: Intrinsically disordered regions can contribute promiscuous interactions to RNP granule assembly. Cell Reports 2018;22:1401-1412.

11 Buchan JR: mRNP granules. Assembly, function, and connections with disease. RNA Biol 2014;11:1019-1030.

-12 Tsekrekou M, Stratigi K, Chatzinikolaou G: The nucleolus: in genome maintenance and repair. Int J Mol Sci 2017;18:E1411.

13 Khanna N, Hu Y, Belmont AS: HSP70 transgene directed motion to nuclear speckles facilitates heat shock activation. Curr Biol 2014; 24:1138-1144.

14 Mahboubi H, Stochaj U: Nucleoli and stress granules: connecting distant relatives. Traffic 2014;15:1179-1193.

15 Nunes VS, Moretti NS: Nuclear subcompartments: an overview. Cell Biol Int 2017;41:2-7.
16 Yamazaki T, Hirose T: The building process of the functional paraspeckle with long noncoding RNAs. Front Biosci (Elite Ed) 2015; 7: $1-41$.

17 Rajgor D, Shanahan CM: RNA granules and cytoskeletal links. Biochem Soc Trans 2014; 42:1206-1210.

18 Singh G, Pratt G, Yeo GW, Moore MJ: The clothes make the mRNA: past and present trends in mRNP fashion. Annu Rev Biochem 2015;84:325-354.

19 Decker CJ, Parker R: P-bodies and stress granules: possible roles in the control of translation and mRNA degradation. Cold Spring Harb Perspect Biol 2012;4:a012286.

20 Hubstenberger A, Courel M, Benard M, Souquere S, Ernoult-Lange M, Chouaib R, Yi Z, Morlot JB, Munier A, Fradet M, Daunesse M, Bertrand E, Pierron G, Mozziconacci J, Kress M, Weil D: P-body purification reveals the condensation of repressed mRNA regulons. Mol Cell 2017;68:144-157.e145.

21 Hoyle NP, Castelli LM, Campbell SG, Holmes LE, Ashe MP: Stress-dependent relocalization of translationally primed mRNPs to cytoplasmic granules that are kinetically and spatially distinct from P-bodies. J Cell Biol 2007;179: 65-74.

22 Khong A, Matheny T, Jain S, Mitchell SF, Wheeler JR, Parker R: The stress granule transcriptome reveals principles of mRNA accumulation in stress granules. Mol Cell 2017;68: 808-820.e805.

23 Leung AKL, Sharp PA: MicroRNA functions in stress responses. Mol Cell 2010;40:205215.

24 Buchan JR, Parker R: Eukaryotic stress granules: the ins and outs of translation. Mol Cell 2009;36:932-941.

25 Moujaber O, Mahboubi H, Kodiha M, Bouttier M, Bednarz K, Bakshi R, White J, Larose L, Colmegna I, Stochaj U: Dissecting the molecular mechanisms that impair stress granule formation in aging cells. Biochim Biophys Acta 2017;1864:475-480.

26 Walter P, Ron D: The unfolded protein response: from stress pathway to homeostatic regulation. Science 2011;334:1081-1086.

27 Zheng D, Chen C-YA, Shyu A-B: Unraveling regulation and new components of human $\mathrm{P}$ bodies through a protein interaction framework and experimental validation. RNA 2011; 17:1619-1634.
28 Eulalio A, Behm-Ansmant I, Izaurralde E: P bodies: at the crossroads of post-transcriptional pathways. Nat Rev Mol Cell Biol 2007; $8: 9-22$.

29 Ayache J, Benard M, Ernoult-Lange M, Minshall N, Standart N, Kress M, Weil D: P-body assembly requires DDX6 repression complexes rather than decay or Ataxin $2 / 2 \mathrm{~L} \mathrm{com}$ plexes. Mol Biol Cell 2015;26:2579-2595.

-30 Zheng D, Ezzeddine N, Chen CY, Zhu W, He $\mathrm{X}$, Shyu AB: Deadenylation is prerequisite for P-body formation and mRNA decay in mammalian cells. J Cell Biol 2008;182:89-101.

- 31 Eulalio A, Behm-Ansmant I, Schweizer D, Izaurralde $\mathrm{E}$ : P-body formation is a consequence, not the cause, of RNA-mediated gene silencing. Mol Cell Biol 2007;27:3970-3981.

32 Patranabis S, Bhattacharyya SN: P-body-induced inactivation of let-7a miRNP prevents the death of growth factor-deprived neuronal cells. FASEB J 2017;32:1493-1509.

33 Herrero AB, Moreno S: Lsm1 promotes genomic stability by controlling histone mRNA decay. EMBO J 2011;30:2008-2018.

34 Loll-Krippleber R, Brown GW: P-body proteins regulate transcriptional rewiring to promote DNA replication stress resistance. Nat Commun 2017;8:558.

35 Jang C, Lahens NF, Hogenesch JB, Sehgal A: Ribosome profiling reveals an important role for translational control in circadian gene expression. Genome Res 2015;25:1836-1847.

-36 Shigeoka T, Jung H, Jung J, Turner-Bridger B, Ohk J, Lin JQ, Amieux PS, Holt CE: Dynamic axonal translation in developing and mature visual circuits. Cell 2016;166:181-192.

37 Graber TE, Freemantle E, Anadolu MN, Hebert-Seropian S, MacAdam RL, Shin U, Hoang HD, Alain T, Lacaille JC, Sossin WS: UPF1 governs synaptic plasticity through Association with a STAU2 RNA granule. J Neurosci 2017;37:9116-9131.

38 El Fatimy R, Davidovic L, Tremblay S, Jaglin X, Dury A, Robert C, De Koninck P, Khandjian EW: Tracking the fragile $\mathrm{X}$ mental retardation protein in a highly ordered neuronal ribonucleoparticles population: a link between stalled polyribosomes and RNA granules. PLoS Genet 2016;12:e1006192.

39 Ouwenga R, Lake AM, O’Brien D, Mogha A, Dani A, Dougherty JD: Transcriptomic analysis of ribosome-bound mRNA in cortical neurites in vivo. J Neurosci 2017;37:8688-8705. 
40 Parchure A, Munson M, Budnik V: Getting mRNA-Containing ribonucleoprotein granules out of a nuclear back door. Neuron 2017; 96:604-615.

41 Maziuk B, Ballance HI, Wolozin B: Dysregulation of RNA binding protein aggregation in neurodegenerative disorders. Front Mol Neurosci 2017;10:89.

-42 Jonson L, Vikesaa J, Krogh A, Nielsen LK, Hansen T, Borup R, Johnsen AH, Christiansen J, Nielsen FC: Molecular composition of IMP1 ribonucleoprotein granules. Mol Cell Proteomics 2007;6:798-811.

43 Moschner K, Sundermann F, Meyer H, da Graca AP, Appel N, Paululat A, Bakota L, Brandt R: RNA protein granules modulate tau isoform expression and induce neuronal sprouting. J Biol Chem 2014;289:16814-16825.

44 Buckingham M, Liu JL: U bodies respond to nutrient stress in Drosophila. Exp Cell Res 2011;317:2835-2844.

-45 Lui J, Castelli LM, Pizzinga M, Simpson CE, Hoyle NP, Bailey KL, Campbell SG, Ashe MP: Granules harboring translationally active mRNAs provide a platform for P-body formation following stress. Cell Rep 2014;9:944-954.

46 Kourtidis A, Necela B, Lin WH, Lu R, Feathers RW, Asmann YW, Thompson EA, Anastasiadis PZ: Cadherin complexes recruit mRNAs and RISC to regulate epithelial cell signaling. J Cell Biol 2017;216:3073-3085.

47 Jourdain AA, Boehm E, Maundrell K, Martinou JC: Mitochondrial RNA granules: compartmentalizing mitochondrial gene expression. J Cell Biol 2016;212:611-614.

48 Zaganelli S, Rebelo-Guiomar P, Maundrell K, Rozanska A, Pierredon S, Powell CA, Jourdain AA, Hulo N, Lightowlers RN, Chrzanowska-Lightowlers ZM, Minczuk M, Marti- nou JC: The pseudouridine synthase RPUSD4 is an essential component of mitochondrial RNA granules. J Biol Chem 2017;292:45194532.

49 Wheeler JR, Matheny T, Jain S, Abrisch R, Parker R: Distinct stages in stress granule assembly and disassembly. Elife 2016;5:e18413.

50 Kedersha N, Ivanov P, Anderson P: Stress granules and cell signaling: more than just a passing phase? Trends Biochem Sci 2013;38: 494-506.

51 Tourriere H, Chebli K, Zekri L, Courselaud B, Blanchard JM, Bertrand E, Tazi J: The RasGAP-associated endoribonuclease G3BP assembles stress granules. J Cell Biol 2003;160: 823-831.

52 Ganassi M, Mateju D, Bigi I, Mediani L, Poser I, Lee HO, Seguin SJ, Morelli FF, Vinet J, Leo G, Pansarasa O, Cereda C, Poletti A, Alberti S, Carra S: A surveillance function of the HSPB8-BAG3-HSP70 Chaperone complex ensures stress granule integrity and dynamism. Mol Cell 2016;63:796-810.

53 Bley N, Lederer M, Pfalz B, Reinke C, Fuchs T, Glass M, Moller B, Huttelmaier S: Stress granules are dispensable for mRNA stabilization during cellular stress. Nucleic Acids Res 2015;43:e26.

54 Lian XJ, Gallouzi IE: Oxidative stress increases the number of stress granules in senescent cells and triggers a rapid decrease in p21waf1/ cip1 translation. J Biol Chem 2009;284:88778887.

55 Zhan Y, Dhaliwal JS, Adjibade P, Uniacke J, Mazroui R, Zerges W: Localized control of oxidized RNA. J Cell Sci 2015;128:4210-4219.

56 Aragon T, van Anken E, Pincus D, Serafimova IM, Korennykh AV, Rubio CA, Walter P: Messenger RNA targeting to endoplasmic re- ticulum stress signalling sites. Nature 2009; 457:736-740.

57 Lechler MC, Crawford ED, Groh N, Widmaier K, Jung R, Kirstein J, Trinidad JC, Burlingame AL, David DC: Reduced insulin/IGF-1 signaling restores the dynamic properties of key stress granule proteins during aging. Cell Reports 2018;18:454-467.

58 Kramer S: RNA in development: how ribonucleoprotein granules regulate the life cycles of pathogenic protozoa. Wiley Interdiscip Rev RNA 2014;5:263-284.

59 Anderson P, Kedersha N, Ivanov P: Stress granules, P-bodies and cancer. Biochim Biophys Acta 2015;1859:861-870.

-60 Bergalet J, Fawal M, Morello D, Espinos E: ALK-mediated post-transcriptional regulation: focus on RNA-binding proteins. Front Biosci (Landmark Ed) 2015;20:1250-1258.

61 Imig J, Kanitz A, Gerber AP: RNA regulons and the RNA-protein interaction network. Biomol Concepts 2012;3:403-414.

-62 Ariumi Y, Kuroki M, Kushima Y, Osugi K, Hijikata M, Maki M, Ikeda M, Kato N: Hepatitis $\mathrm{C}$ virus hijacks $\mathrm{P}$-body and stress granule components around lipid droplets. J Virol 2011;85:6882-6892.

63 Pager CT, Schutz S, Abraham TM, Luo G, Sarnow P: Modulation of hepatitis $C$ virus RNA abundance and virus release by dispersion of processing bodies and enrichment of stress granules. Virology 2013;435:472-484.

-64 Martínez JP, Pérez-Vilaró G, Muthukumar Y, Scheller N, Hirsch T, Diestel R, Steinmetz H, Jansen R, Frank R, Sasse F, Meyerhans A, Díez $\mathrm{J}$ : Screening of small molecules affecting mammalian P-body assembly uncovers links with diverse intracellular processes and organelle physiology. RNA Biol 2013;10:1661-1669. 